\title{
Pseudoelastic behavior of hypostoichiometric NiAl alloys: A simple model
}

\author{
Y. Y. Ye* \\ Ames Laboratory and Department of Physics and Astronomy, Iowa State University, Ames, Iowa 50011 \\ and Metals and Ceramics Division, Oak Ridge National Laboratory, Oak Ridge, Tennessee 37831-6115 \\ C. T. Chan, K. M. Ho, and C. Z. Wang \\ Ames Laboratory and Department of Physics and Astronomy, Iowa State University, Ames, Iowa 50011
}

(Received 24 February 1993; revised manuscript received 8 November 1993)

\begin{abstract}
We studied the pseudoelastic behavior of hypostoichiometric $\mathrm{NiAl}$ alloys using a very simple model, with interplanar interaction parameters extracted from recent neutron-scattering experiments. The behavior of the system under shear is studied numerically both in the static (quasiequilibrium) limit and with molecular dynamic simulations. Our results indicate that anomalous phonon softening can facilitate the nucleation of the martensitic phase near boundaries or defects as the system approaches the transformation temperature. For our simple model, we found that the critical stress for the nucleation of the martensitic phase is reduced by almost a factor of 2 as a result of anomalous phonon softening near the martensitic transformation temperature.
\end{abstract}

\section{INTRODUCTION}

The subject of martensitic phase transformation is one of considerable scientific as well as technological interest. Among the martensitic materials, the "shape-memory" alloys (e.g., NiTi, NiAl) have exotic elastic properties near the martensitic transformation temperature $\left(M_{s}\right)$ : they can be subjected to phenomenal strains but can recover completely either when the loading stress is released (pseudoelastic effect) or by heating the sample (thermoelastic effect). ${ }^{1}$ In many of these systems, partial softening of anomalous phonon modes has been reported as one approaches $M_{s}{ }^{2-4}$ In this article, we show that many of these interesting and unusual elastic behaviors can be simulated and discussed in terms of a very simple model which includes the anomalous phonon spectrum as a key ingredient. ${ }^{5}$ Our results indicate that anomalous phonon softening can facilitate the nucleation of the martensitic phase near boundaries or defects as the system approaches the transformation temperature, causing a reduction of the critical stress required to nucleate the martensitic phase by almost a factor of 2 . We also found that, because of the presence of strong anharmonic effects and the heterogeneous nature of the nucleation process, phonon softening at an arbitrary wave vector can assist the nucleation of a martensitic phase which does not contain the displacements of the softening phonon.

We choose the $\mathrm{NiAl}$ alloys as a prototype to study these unusual elastic behaviors because detailed studies ${ }^{6,7}$ of the anomalous phonon behavior in these alloys have been performed in connection with the martensitic transformation. The phonon dispersion curves along the [110] direction have been measured for the transverse-acoustic (TA) mode at several temperatures. As the temperature is lowered towards $M_{s}$, a dip appears in the dispersion curve near $q=0.16[110]$. Such a dip is usually referred to as a phonon anomaly. If the phonon frequency goes all the way to zero as the temperature decreases, it means that there is an unstable mode in the system and the system will undergo a second-order transformation to a new structure, as determined by the wave vector that goes "soft" and the anharmonicity of the interactions. For the case of hypostoichiometric $\mathrm{NiAl}$ alloys with $\mathrm{Ni}$ concentrations in excess of $63 \%,{ }^{8}$ the crystal transforms to a close-packed structure before the "dip" goes to zero. Nevertheless, we will see that the phonon anomaly still plays an important role in the martensitic transformation and the pseudoelastic properties of this material.

\section{THE MODEL}

We will study this problem by a very simple model which treats the system as a stack of rigid (110) planes connected by a specified set of interplanar interactions. Although this model is certainly oversimplified, we believe that it can capture and highlight the essence of the problem. In the $B 2$ structure, the shear-and-shuffle displacements of the (110) planes in the lattice along the [110] direction are important for the martensitic transformation from the parent phase to various close-packed structures. ${ }^{9-11}$ The experimental phonon dispersion data allow us to extract the interplanar interactions: the Fourier transform of the square of the phonon frequencies of the TA[110] branch as a function of wave vector will give us the "Born-von-Karman" force constants acting between various (110) planes in the crystal. However, while the phonon dispersion curves can provide information relevant to small displacements, a completely harmonic model is not adequate for describing elastic response phenomena which involve large strains. Thus we write the interplanar interaction between one plane and its $p$ th neighbor in the form

$$
E_{p}=-W_{p} \cos \left[\frac{2 \pi}{b} x_{p}\right]
$$



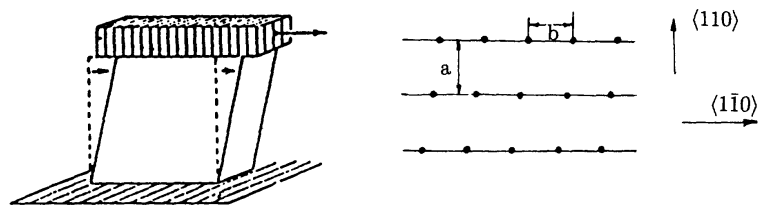

FIG. 1. Schematic pictures illustrating the interplanar force model. See text for discussions.

where $b(=\sqrt{2} \times 2.858 \AA)$ is the distance in which the system repeats itself in the [110] direction and $x_{p}$ is the displacement of the $n$th plane relative to the $(n+p)$ th plane as depicted pictorially in Fig. 1. Equation (1) is the simplest form which guarantees that the interaction satisfies the condition of translational periodicity: a necessary condition for any interaction that describes large shear displacements. Expanding Eq. (1) to second order readily gives the phonon dispersion of this model:

$$
[\hbar \omega(q)]^{2}=\sum_{p>0} 2 C_{p}[1-\cos (q p a)]
$$

where $a$ is the distance between neighboring (110) planes and $C_{p}=W_{p} \frac{\hbar^{2}}{M}\left(\frac{2 \pi}{b}\right)^{2} . M$ is the atomic mass of the system. In the present case, we choose $M$ to be the average atomic mass of $\mathrm{Ni}$ and $\mathrm{Al}$ weighted by their individual concentrations, which is about 46.8 atomic mass units. (A further refinement of the model can take into account the variation of $M$ in the crystal.) Fitting the phonon curves with Eq. (2) will give the interplanar interaction parameters for the model described in Eq. (1). For the case of $\mathrm{NiAl}$, we found that the force constants are fairly long ranged. This is a direct consequence of the existence of the phonon "anomaly." Interactions up to eight nearest-neighbor planes are needed at the lowest temperature to reproduce faithfully the phonon dispersion curves in regions where the phonon dip is most pronounced. In Table I, we list the interplanar parameters $C_{p}$, which give the phonon frequencies in $(\mathrm{meV})^{2}$ when substituted into Eq. (2). Since the phonon dispersion curves are measured at three different temperatures, the present procedures give three sets of parameters, each describing the effective interactions, including entropy effects, at each temperature.

\section{QUASISTATIC SIMULATIONS}

Once the coupling parameters are determined, we can perform numerical simulations to explore the elastic be- havior of our model NiAl. It is instructive to examine the behavior of the model system under a uniform strain. For the lower temperatures ( 150 and $85 \mathrm{~K})$, we found that the energy change per atom of the system under a uniform shear is far from the quadratic behavior expected for a linear-response system. There exists a second local minimum, which corresponds to a metastable state that becomes increasingly more stable as the temperature is lowered. This correlates nicely with the phonon dispersion curves where the phonon anomaly becomes more pronounced as the temperature is lowered.

The stress-strain $(\sigma-\epsilon)$ behavior of the NiAl model system is then studied numerically under loading (increasing stress) and unloading (decreasing stress) situations. Our model system consists of a slab of 100 layers of (110) planes with periodic boundary conditions imposed so that the system acquires a zigzag twinned pattern when the 100-layered slab is sheared. We have also repeated the whole calculation with different boundary conditions corresponding to a finite 100-layer slab and the results are essentially the same, except that the yield stresses are slightly lowered. We will first present results in the quasistatic (quasiequilibrium) limit. In this limit, equal and opposite small incremental displacements (shears) are imposed on the boundary layers. With the displacements of the boundary layers fixed, the other layers are relaxed by a steepest-descent algorithm until a new equilibrium position is obtained. After reaching equilibrium, the forces acting on the atoms in boundary layers are recorded. Stress-strain curves are obtained in this manner for three different temperatures as shown in Fig. 2. In this figure, the stress-strain relations obtained for increasing strain (loading) are indicated by circles. The data obtained during the unloading process are indicated by crosses. It is interesting to see that the $\sigma-\epsilon$ curves reproduce all the salient features of the experimental $\sigma-\epsilon$ curves of a typical pseudoelastic or thermoelastic alloy. ${ }^{12}$

Upon loading, the system behaves elastically for small strains until the stress exceeds the yield stress $\left(\sigma_{u}\right)$. When the system is strained beyond this point, there is a sudden drop in the stress to a constant-stress plateau where the strain of the system increases while the stress remains at a constant value which we label the transformation stress $\left(\sigma_{t}\right)$. When the strain reaches $\sim 30 \%$, the stress again increases with strain, leading to a stressstrain curve with two "elastic" regimes separated by a region where strain increases at constant stress. Upon unloading, the system completely recovers (pseudoelastic) at higher temperatures. At lower temperatures, residual

TABLE I. Three sets of the interplanar parameters, each describing the effective interactions at each temperature. See text for definition.

\begin{tabular}{crrrr}
\hline \hline $85 \mathrm{~K}$ & 25.90652 & -3.77912 & -4.40312 & 1.72748 \\
& 0.74508 & -0.04872 & 0.43204 & -0.52400 \\
$150 \mathrm{~K}$ & 25.84700 & -3.75079 & -4.25987 & 1.79874 \\
& 0.74170 & -0.14811 & 0.39906 & -0.46753 \\
$290 \mathrm{~K}$ & 24.23627 & 0.39080 & -6.59759 & 1.29493 \\
& 2.39755 & -1.12725 & &
\end{tabular}


strain remains upon unloading, but complete recovery can be obtained by further relaxation using the effective interaction parameters of higher temperatures, corresponding to complete strain recovery upon heating the system (thermoelastic behavior).

In Fig. 3, we display the displacements of the individual layers in our system at various stages during the loading-unloading process for the simulation with the $T=290 \mathrm{~K}$ parameters. Initially, for small strains upon loading, the system behaves normally and the strain is distributed uniformly across the system [configurations $A$ and $B$ in Fig. 3(a)]. Beyond the yield stress, in the plateau region, we find that the system breaks up into two regions [configurations $\mathrm{C}$ and $\mathrm{D}$ in Fig. 3(a)], one with small strain and one with large strain, and the system can increase its overall strain at constant stress by changing the ratio of the high-strain portion to the lowstrain portion. Beyond the plateau, the strain is again distributed uniformly across the system [configuration $\mathrm{E}$ in Fig. 3(a)]. Figure 3(b) shows the strain profiles of the system upon unloading, which is basically the reverse of the loading behavior. Examination of our results reveals that the response of the system upon loading is a manifestation of a stress-induced martensitic transformation. The two elastic regions, one at small $(\epsilon<8 \%)$ and the other at larger $(\epsilon \sim 30 \%)$ strain, correspond to the elastic properties of the parent crystal and its stress-induced martensitic phase respectively, whereas the plateau in the $\sigma-\epsilon$ curve represents a transformation process in which the system contains a mixture of both phases. At a lower temperature (e.g., $85 \mathrm{~K}$ ), the residual strains remain upon unloading since the stress-induced marten-

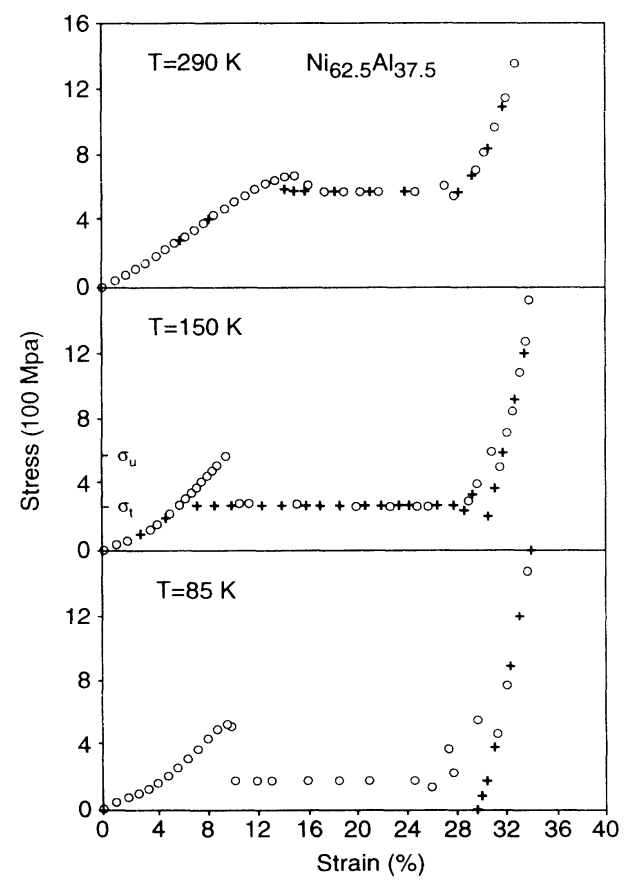

FIG. 2. Theoretical stress-strain relationships at three temperatures as found from the numerical simulation. Open circles and crosses correspond to loading and unloading respectively. sitic phase is metastable, but it becomes unstable if the system is heated to higher temperatures, leading to complete recovery of the parent phase.

In "normal" materials, plastic deformation beyond the elastic limit occurs through the irreversible movements of dislocations. Since dislocations are not included in our model, the pseudoelastic behavior we described above can only occur when the critical stress to cause a stressinduced martensitic transformation $\sigma_{u}$ lies below the critical flow stress for dislocations in the material. To get an idea about the magnitude of $\sigma_{u}$, we compare it with the spinodal stress $\left(\sigma_{s}\right)$, given by the slope of the energy-
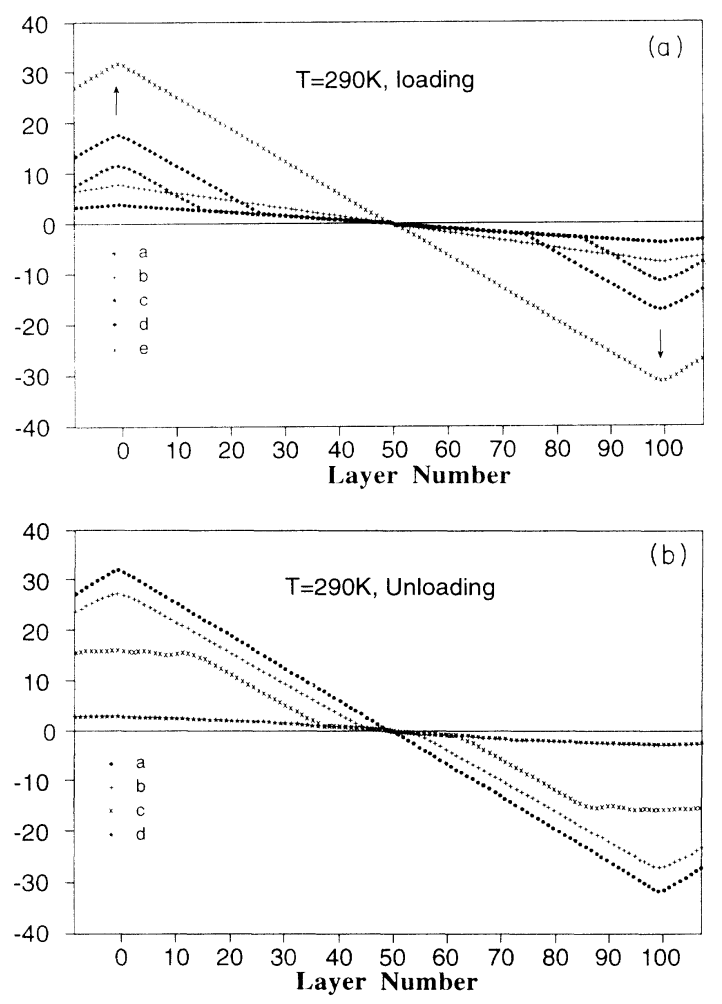

FIG. 3. Schematic pictures showing the strain profiles for the model system upon (a) loading and (b) unloading, at 290 $\mathrm{K}$. Each symbol represents the displacement of one layer from the unstrained position, and if the profile is a straight line, the strain is uniformly distributed. For loading, the strains at the boundary layers for the configurations $\mathrm{A}, \mathrm{B}, \mathrm{C}, \mathrm{D}, \mathrm{E}$ are respectively $4 \%, 7.5 \%, 11 \%, 16 \%$, and $31 \%$, while for unloading, the strains at the boundary layers for the configurations A,B,C,D are $31 \%, 27 \%, 16 \%$, and $3 \%$ respectively. The 0 th and 100 th layers are the boundary layers, with periodic boundary condition. Upon loading and under small stress [configurations A,B in Fig. 3(a)], the strain is essentially uniformly distributed. Above a critical stress, the system breaks up into two portions (configurations C,D), one with larger strain than the other. The system eventually reaches a second elastic region (configuration E) corresponding to a daughter phase which is metastable, but has a lower enthalpy under external stress. Upon unloading [Fig. 3(b)], there are also two elastic regions (A,B correspond to the daughter phase and D,E correspond to the parent phase under external stress), separated by a regime of mixed phases (configuration $\mathrm{C}$ ). 
strain curve at the inflection point when the system is subjected to a homogeneous strain. Under such a stress, the parent phase becomes unstable towards spontaneous transformation to the daughter phase at all points in the system. We found that while the $\sigma_{s}$ values are very similar for the three different temperatures, the yield stresses $\left(\sigma_{u}\right)$ obtained from our simulations show a substantial drop with decreasing temperatures. With the $290-\mathrm{K}$ parameters, when the phonon anomaly is weak, $\sigma_{s}(\sim 700$ $\mathrm{MPa})$ is about equal to $\sigma_{u}(\sim 680 \mathrm{MPa})$. This is similar to the behavior observed in more detailed molecular dynamics simulations using short-ranged interatomic interactions. ${ }^{13}$ In these cases, since $\sigma_{u}$ for the nucleation of the martensitic phase is so close to the stress when the whole system becomes unstable under a homogeneous strain, it is obvious that the stress-induced phase-transition mechanism will be no match for the dislocation-flow plastic deformation process and thus we should observe no pseudoelastic effects for the real crystal system when dislocations are present.

At lower temperatures, when the phonon anomaly becomes more pronounced, the system exhibits pseudoelastic behavior at a $\sigma_{u}$ considerably lower than $\sigma_{s}$ (at 85 $\mathrm{K}, \sigma_{u}$ is approximately $60 \%$ of $\sigma_{s}$ ). The existence of a strong phonon anomaly in the TA[110] phonon branch is strongly correlated with a substantial lowering of $\sigma_{u}$ which will favor pseudoelastic deformation over plastic deformation involving dislocation-flow processes.

To understand the mechanism for the lowering of $\sigma_{u}$, we observed the deformation of the system during loading at $85 \mathrm{~K}$ (see Fig. 4) and contrast the behavior with that at $290 \mathrm{~K}$. We found that, at $85 \mathrm{~K}$, when the phonon anomaly is very pronounced, the distribution of the strain in the system is not as uniform as in the case of $290 \mathrm{~K}$ when the phonon anomaly is weak. As shown in Fig. $4(\mathrm{a})$, even at stresses below $\sigma_{u}$, the displacement pattern of the system has an oscillatory component near the boundary where the stress is applied. The oscillatory component decays away from the boundary and it resembles a frozen-in displacement of the phonon mode that has a wave vector in the dip of the phonon spectrum. Thus, the local strain in the layers next to the boundary will approach the strain of the metastable martensitic phase at stresses far below the spinodal stress (which is derived under the assumption that the system is uniformly strained). When, at $\sigma_{u}$, the local strain reaches the strain of the martensitic phase, an embryo of the daughter phase is formed at the boundary and the system will become unstable towards a rapid growth of the daughter phase into the parent phase. In Fig. 4(b), we indicate the evolution of the system (following a steepestdescent algorithm as described above), when we start with the system just below $\sigma_{u}$ and increase the displacements of the boundary layers by a very small amount. This sequence marks the transition from the elastic region to the plateau region of the stress-strain curve; at the end of the sequence, when the system is once again in equilibrium, the stress at the boundary has decreased from $\sigma_{u}$ to the plateau value of $\sigma_{t}$.

The existence of the stress-induced phonon oscillation near the boundary can be understood qualitatively from a harmonic analysis. If we look at the problem in reciprocal space, the linear response of the system to an external force $F(q)$ is given by $u(q)=F(q) /\left[M \omega^{2}(q)\right]$. If the external stress is applied only to the boundary layers, $F(q)$ will be fairly constant for a large range of wave vectors $q$. Thus the dip in $\omega(q)$ will enhance the amplitudes of the response $u(q)$ for wave vectors in the region of the phonon anomaly. Within the harmonic regime, such "modulated lattice relaxation" has been discussed by Yamada, ${ }^{14}$ and has been related by Yamada to premartensitic behaviors in $\mathrm{TiNi}(\mathrm{Fe})$ alloys, especially the zone dependence of the incommensurability of $\mathrm{x}$-ray satellite reflections. ${ }^{15}$ Since $\omega(q)$ is still nonzero, it costs energy to induce these static displacements far inside the bulk of the system and thus they will be largest near the boundaries. However, the above discussion offers only an oversimplified description because, as the shear displacement increases, anharmonic effects have to be taken into consideration.

An important effect of anharmonicity is that, al-
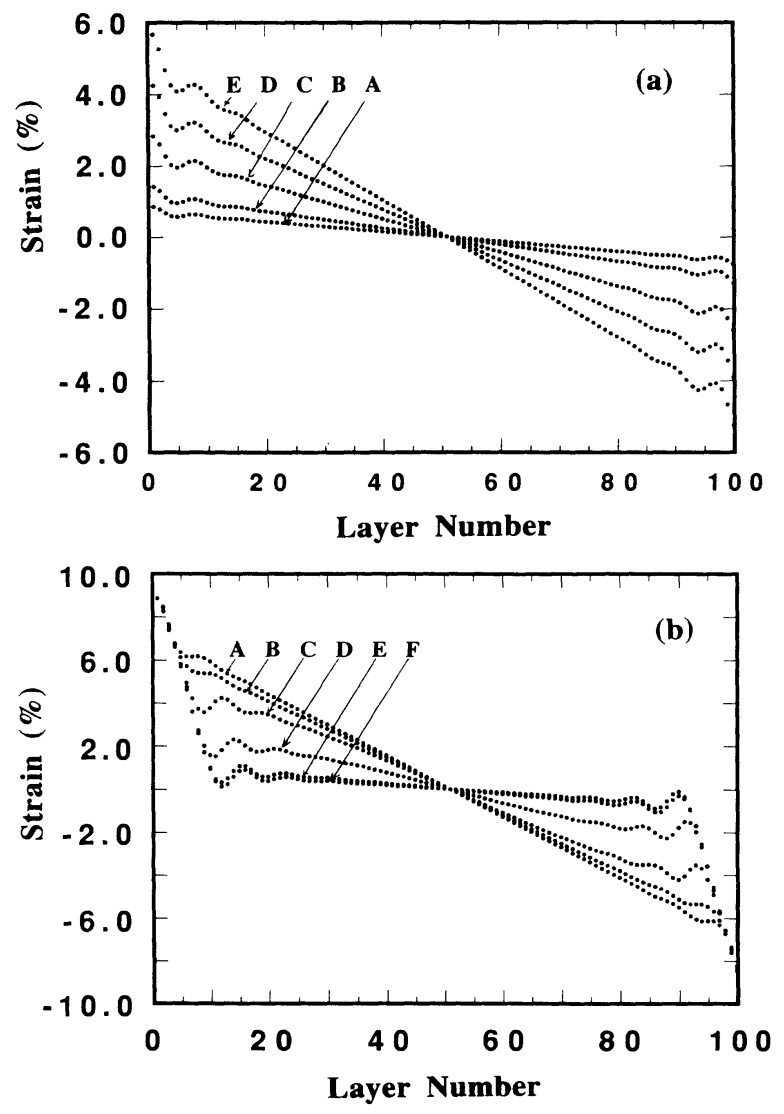

FIG. 4. Strain profiles (displacements of the individual layers plotted against the layer number) of the model system at $85 \mathrm{~K}$ upon loading. Note the frozen-phonon-like displacements near the boundary layers, which should be contrasted with the linear profiles at $290 \mathrm{~K}$ (except in the mixed-phase regions). Fig. 4(a) shows the profiles when the stress is below the critical stress and Fig. 4(b) shows the profiles during the transformation from the linear to the plateau region of the stress-strain curve when the system is strained slightly beyond the yield point. See text for more discussions. The strains on the boundary layers increase with the alphabetical order of the configurations. 
though the anomalous phonon helps the nucleation of the martensitic phase, its displacement need not be retained in the final bulk martensitic crystal. For example, in the present case, while the anomalous phonon has nonzero wave vector corresponding to an oscillatory displacement of the layers, the final nucleated phase consists only of a homogeneous shearing of the parent phase with no oscillatory components.

In our simulations, we have not included the effects of defects which could further lower the stress necessary for nucleation. The $\sigma_{u}$ obtained from our calculations are considerably bigger than the experimental observed yield stress, ${ }^{16}$ partly because we have allowed for only one degree of freedom in the transformation. It also indicates the importance of defects as well as intrinsic interactions of the material for a complete quantitative understanding of the pseudoelastic problem.

In the unloading process, we let the system relax under the steepest-descent algorithm for a few steps, then we freeze the positions of the boundary layers, let the rest of the system relax to equilibrium, and record the stress at the boundary layers. We note that during the unloading process, in the region near the beginning and the end of the plateau region, there are reproducible oscillations in the calculated stress of the system which can be related to the squeezing out of the last few layers of the parent or the daughter phase to get the system back into a uniformstrain state.

In the quasistatic limit, the effect of temperature enters implicitly through the effective interactions that are derived from phonon dispersion measurements at a particular temperature. Such a description ignores fluctuation effects at finite temperatures. To see how thermal fluctuations influence our results, we modified the aforementioned method as follows: to find out the equilibrium configuration for a certain fixed displacement of the boundary layers, instead of starting with the last equilibrium configuration as described above, we start with a configuration in which we disrupt the last equilibrium configuration with random displacements such that the overall potential energy of the system increases by approximately $\frac{1}{2} k_{B} T$ per atom. We found that the two methods give essentially the same results. The insensitivity of our results towards thermal fluctuations indicates that, although we employ a one-dimensional model in our calculations, our conclusions may also be applicable in higher dimensions.

\section{MOLECULAR DYNAMICS SIMULATION}

In a further attempt to study the effects of thermal fluctuations, we studied the stress-strain behavior of our model system using molecular dynamical simulations. There are two difficulties associated with our simple model. First, our model is effectively a one-dimensional model and thus fluctuation effects are greatly exaggerated in molecular dynamics studies. Such a model cannot even have true long-range order at finite-temperatures in the thermodynamic limit. This is an intrinsic shortcoming of our model. Fortunately, we are considering
TABLE II. Modified coupling parameters at $85 \mathrm{~K}$.

\begin{tabular}{rrrrr}
\hline \hline $85 \mathrm{~K}$ & 27.63093 & -4.23127 & -5.80001 & 2.41038 \\
& 1.17749 & -0.26535 & 0.67947 & -0.78641 \\
\hline
\end{tabular}

a relatively small finite-size system in which the longwavelength fluctuations are suppressed by imposing periodic boundary conditions. We will only present results corresponding to the lowest temperature $(85 \mathrm{~K})$ where the fluctuation effects are still tolerable. The second difficulty is a technical one. Our interaction parameters are obtained from experimental measurements, which already contain temperature effects. If we perform molecular dynamics simulations with these interactions, there will be a double counting of the entropy effects. To be more specific, if we use the interlayer interactions as is, and obtain the phonon frequencies at a particular temperature (using velocity-velocity autocorrelation functions to take into account anharmonic effects), we will obtain anharmonic shifts of the phonon frequencies away from the fitted values. To overcome this inconsistency, we adopted the following procedure. Instead of performing one single fit (essentially a Born-von-Karman transform), we modified our coupling parameters iteratively until the phonon frequencies obtained at a finite temperature (as found from the position-position or velocity-velocity correlation function) agree with those of the experimental results at the same temperature. Coupling parameters determined via this procedure are listed in Table II.

With these two points in mind, we performed molecular dynamics simulations at a temperature of $85 \mathrm{~K}$. The temperature is controlled by velocity rescaling and thermalization of the system was done by running the system (without external stress) for 10000 time steps before loading begins. Our model system is again the same periodic system consisting of 50 layers of model NiAl. Instead of imposing strains as we have done for the quasistatic case, we now impose equal and opposite incremental stresses on the boundary layers, and measure the strains on the boundary layers. Each molecular dynamics time step corresponds to about $3.7 \mathrm{fs}$, and after each time step, we increase the stress on the boundary layers by about $64 \mathrm{~N} / \mathrm{m}^{2}$. The whole loading process is accomplished in about $30 \mathrm{~ns}$. Such a rate of stress increase is slow enough so that the layers in the interior have time to respond, since elastic waves takes about $13 \mathrm{ps}$ to travel the distance of 50 layers in our model system. If we increase the loading rate significantly, the boundary layers are found to detach from the interior, signaling that the interior layers do not have time to respond to the increase of stress in the boundary. On the other hand, if we reduce the rate of stress increase significantly, we find that the system is disordered by thermal fluctuations before the loading is completed. The resulting $\sigma-\epsilon$ curve is shown in Fig. 5. The noise in the curve is an intrinsic problem of a one-dimensional model, as mentioned above. When the stress is small, we again observe a linear regime where the average configuration is essentially a homogeneously strained system. Further increases in 


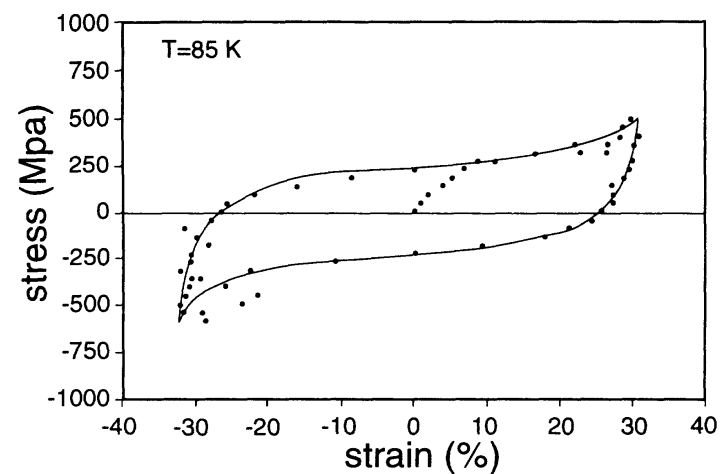

FIG. 5. Stress-strain relationship for the system at $85 \mathrm{~K}$ as found from molecular dynamics simulations. The solid line is drawn as a guide to the eye.

stress will nucleate the daughter phase, where the strain increases rapidly at nearly constant stress until the entire system is transformed into the new phase. At this point, we reverse the increment of the stress on the boundary layer and return the system to zero stress. We find that the strain of the system remains stuck at a finite value corresponding to the daughter phase. We have to reverse the stress before we can bring the system back to zero strain and the whole system displays a classic hysteresis behavior. The energy represented by the area enclosed by the hysteresis loop is essentially the energy needed to create the interface between the parent and daughter phase, which is estimated to be about $80 \mathrm{~mJ} / \mathrm{m}^{2}$ from the area of the hysteresis loop. This should of course be taken as an order of magnitude estimate, given the simplicity of the model. In summary, all the essential features observed in the quasistatic simulations are also observed in the molecular dynamics simulation, except that here (i) fluctuation effects are overemphasized, and (ii) hysteresis phenomena are observed.

\section{CONCLUSION}

The pseudoelastic behavior of hypostoichiometric NiAl alloys is studied using simulation techniques. Using a very simple, effectively one-dimensional model, with parameters derived from experimental phonon measurements, the calculated stress-strain behavior reproduces the salient features observed experimentally for pseudoelastic systems. Detailed analysis of our results shows that phonon anomalies facilitate the nucleation of the martensitic phase near boundaries or defects as the system approaches the transformation temperature by reducing the critical nucleation stress. We also found that, because of anharmonic effects and the heterogeneous nature of the nucleation process, a softening phonon can help in the nucleation of a martensitic phase even though the final nucleated phase does not retain the displacements of the anomalous phonon.

\section{ACKNOWLEDGMENTS}

We thank Dr. M. H. Yoo for helpful suggestions. Ames Laboratory is operated for U.S. Department of Energy by Iowa State University under Contract No. W-7405ENG-82. This work was supported by the Director of Energy Research, Office of Basic Energy Sciences, including a grant of computer time on the Cray computers at the National Energy Research Supercomputer Center at Livermore.
${ }^{*}$ Permanent address: Department of Physics, Wuhan University, Hubei,Wuhan, PRC.

${ }^{1}$ K. Otsuka and K. Shimizu, Int. Met. Rev. 31, 93 (1986); Met. Forum 4, 142 (1981).

${ }^{2}$ S.K. Satija, S.M. Shapiro, M.B. Salamon, and C.M. Wayman, Phys. Rev. B 29, 6031 (1984).

${ }^{3}$ H. Teitze, M. Mullner, and B. Renker, J. Phys. C 17, L529 (1989).

${ }^{4}$ M. Mori, Y. Yamada, and G. Shirane, Solid State Commun. 17, 127 (1975).

${ }^{5}$ Part of this work has been published in a shorter form in Y.Y. Ye, C.T. Chan, and K.M. Ho, Phys. Rev. Lett. 66, 2018 (1991).

${ }^{6}$ S.M. Shapiro, B.X. Yang, G. Shirane, Y. Noda, and L.E. Tanner, Phys. Rev. Lett. 62, 1298 (1989).

${ }^{7}$ S.M. Shapiro, J.Z. Larese, Y. Noda, S.C. Moss, and L.E. Tanner, Phys. Rev. Lett. 57, 3199 (1986).

${ }^{8}$ D. Schryvers and L.E. Tanner, Ultramicroscopy 32, 241
(1990).

${ }^{9}$ F. Reynaud, Scr. Metall. 11, 765 (1977).

${ }^{10}$ K. Enami, A. Nagasawa, and S. Nenno, Scr. Metall. 12, 223 (1978).

${ }^{11}$ V.V. Martynov, K. Enami, L.G. Khandros, S. Nenno, and A.V. Tkachenko, Phys. Met. Metall. 55(5), 136 (1983); Scr. Metall. 17, 1167 (1983).

${ }^{12}$ L.A. Shepard, in Shape Memory Effects in Alloys, edited by J. Perkins (Plenum Press, New York, 1975), p. 423.

${ }^{13}$ D. Kim, P.C. Clapp, and J.A. Rifkin, in High-Temperature Ordered Intermetallic Alloys IV, edited by L. Johnson, D. P. Pope, and J.O. Stiegler, MRS Symposia Proceedings No. 213 (Materials Research Society, Pittsburgh, 1990), p. 249.

${ }^{14}$ Y. Yamada, Met. Trans. A 19A, 777 (1988).

${ }^{15}$ S.M. Shapiro, Y. Noda, Y. Fujii, and Y. Yamada, Phys. Rev. B 30, 4314 (1984).

${ }^{16}$ L.E. Tanner (private communication). 